\title{
Posterior Lumbar Interbody Fusion Using an Unilateral Cage: A Prospective Study of Clinical Outcome and Stability
}

\author{
Seok Ki Lee ${ }^{1}$, Seok Won $\mathrm{Kim}^{2}$, Chang II Ju², Sung Myung Lee ${ }^{2}$, Myung Hoon $\mathrm{Kim}^{2}$ \\ Departments of ${ }^{1}$ Thoracic Surgery, ${ }^{2}$ Neurosurgery, College of Medicine, Chosun University, Gwangju, Korea
}

Objective: The purpose of this study was to evaluate the clinical and radiological results of instrumented posterior lumbar interbody fusion (PLIF) using an unilateral cage.

Methods: Seventeen patients with unilateral radiculopathy who underwent bilateral percutaneous screw fixation with a single fusion cage inserted on the symptomatic side for treatment of focal degenerative lumbar spine disease were prospectively enrolled in this study. Their clinical results, radiological parameters, and related complications were assessed 10 days, 3 months, and 12 months postoperatively.

Results: There was no pseudarthrosis, instrumented fusion failure, significant cage subsidence, or retropulsion in any patient. The surgery restored the disc space height and maintained it as of 12 months postoperatively and did not exacerbate the lumbar lordotic and scoliotic angles. All patients had excellent or good outcomes according to the modified MacNab's criteria. The mean pain score according to the visual analogue scale was 7.5 preoperatively but had improved to 2.5 when reassessed 3 months postoperatively. The improvement was maintained as of 12 months postoperatively.

Conclusion: In cases of uncomplicated unilateral radiculopathy, PLIF using a single cage can be an effective and safe procedure with the advantage of preserving the posterior elements of the contralateral side. A shorter operative time and greater cost-effectiveness than for PLIF using bilateral cages can be expected.

Key Words: Spine $\cdot$ Fusion $\cdot$ Cage $\cdot$ Unilateral

\section{INTRODUCTION}

Posterior lumbar interbody fusion (PLIF) is a widely performed surgical procedure that alleviates pain and spinal instability resulting from various spinal diseases while restoring disc height and supporting the anterior and middle columns, ${ }^{3,8}$. In particular, PLIF involving the insertion of bilateral interbody cages has been a standard treatment that predictably restores segmental alignment and balance and achieves successful fusion in nearly $100 \%{ }^{6}$. However, only an unilateral cage should be inserted in the presence of certain conditions, such as a narrow disc space, nerve root anomalies that may

- Received: April 30, 2014 • Revised: May 30, 2014

- Accepted: June 2, 2014

Corresponding Author: Myung Hoon Kim, MD, PhD

Department of Neurosurgery, School of Medicine, Chosun University,

365 Pilmun-daero, Dong-gu, Gwangju 501-717, Korea

Tel: +82-62-220-3126, Fax: +82-62-227-4575

E-mail: ns64902@hanmail.net/chosunns@chosun.ac.kr

(2)This is an Open Access article distributed under the terms of the Creative

Commons Attribution Non-Commercial License (http://creativecommons.org/

licenses/by-nc/3.0/) which permits unrestricted non-commercial use, distribution,

and reproduction in any medium, provided the original work is properly cited. block access to the disc space, or epidural scarring ${ }^{4}$. The necessity of PLIF using a unilateral cage in such cases raises the question of whether this procedure can provide acceptable balance, clinical outcome, and fusion. If the use of a unilateral cage proved not to diminish the clinical success rate, it could be applied routinely to reduce the surgical time, blood loss, and risk of neural injury. However, to date there have been few prospective studies of the clinical outcome and radiological results of PLIF involving unilateral cage insertion in patients without the aforementioned special circumstances. The purpose of this study was to evaluate the efficacy and safety of PLIF involving unilateral cage insertion in patients with unilateral radiculopathy. The study hypothesis was that unilateral-cage PLIF does not lower the fusion rate or aggravate scoliotic deformity.

\section{MATERIALS AND METHODS}

From January 2010 to January 2012, 17 patients (11 men and 6 women) with unilateral radiculopathy were enrolled in this study. Sufficient explanation of the procedure was provided and consent thereto obtained before surgery in each case. The inclusion criteria were limited to (1) unilateral radi- 

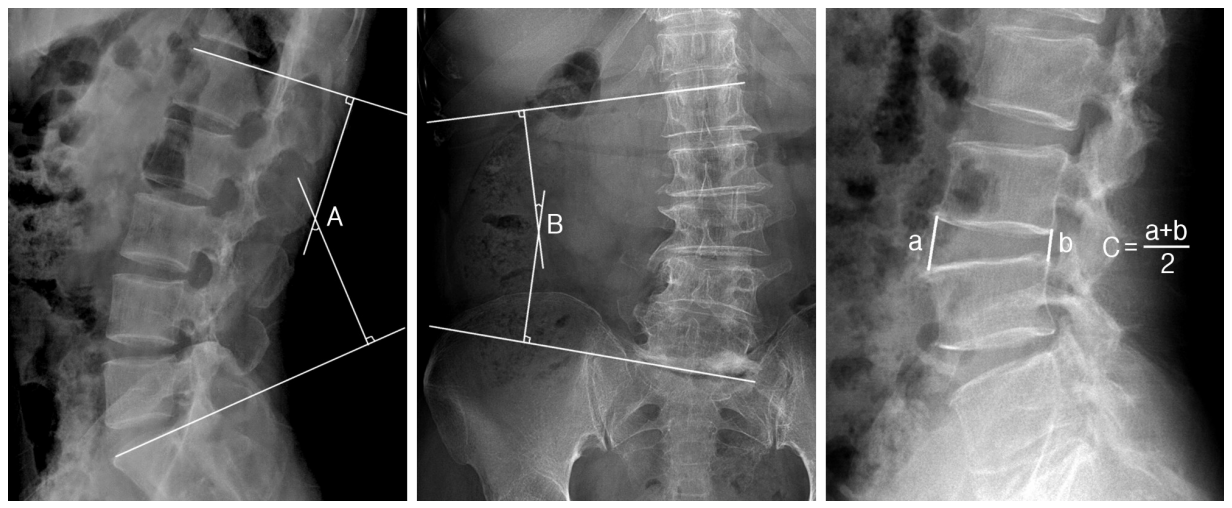

Fig. 1. Cobb method for measurement of the scoliotic angle (A), lordotic angle (B), and height of the disc space $(C)$.

culopathy caused by foraminal stenosis or degenerative disc disease such as upper lumbar disc herniation or recurrent disc herniation and (2) a non-osteoporotic spine (T-score by bone mineral densitometry $>-1.5$ ). Patients with conditions requiring bilateral cage insertion, such as bilateral radiculopathy, significant instability, or spondylolisthesis, and those with spinal osteoporosis were excluded from the study.

\section{Surgical Procedure}

All patients underwent unilateral cage insertion and percutaneous pedicle screw fixation. Complete unilateral facetectomy, hemilaminectomy, and disc space preparation with entire endplate curettage for unilateral cage insertion were performed on the symptomatic side. Then, the end plates of the central portion of the disc space were curetted meticulously, morselized bone graft material obtained from the laminectomy and facetectomy was packed into the cage, and the disc space was filled as compactly as possible with remnant bone chips and allograft bone chips. After cage insertion, a percutaneous transpedicular screw system was used to insert the screws applying compressive force (Clix ${ }^{\circledR}$, Synthes, Switzerland). The patients were instructed to wear a thoraco-lumbo-sacral orthosis (TLSO) when ambulating for 3 months after surgery. All individuals underwent physical and radiological examination preoperatively, 10 days postoperatively, and 3 and 12 months postoperatively. Standing anteroposterior and lateral radiographs including flexion and extension views were obtained and analyzed. Fusion failure was defined as the presence on anteroposterior or lateral radiographs of a definite radiolucent line around a cage or pedicle screw or more than $5^{\circ}$ of motion on lateral flexion-extension radiographs. The height of the intervertebral disc space was calculated as the mean of the sum of the vertical distances between the anterior and posterior edges of the vertebral endplates. The lumbar lordotic angle and scoliotic angle were measured using the Cobb method (Fig. 1). The demographic data, clinical outcomes, fusion success, and related complications were analyzed.

\section{Safety and Outcome Evaluation}

The visual analogue scale (VAS) pain scores were evaluated at the above-mentioned time points. At the 12-month postoperative examination, the patients were evaluated according to a modified version of MacNab's criteria for characterizing clinical outcomes after spinal surgery.

\section{Statistical Analysis}

Statistical analysis was performed using SAS 6.12 (SAS Institute, Inc., Cary, NC, USA). Mean values (MV) and standard deviations (SD) were calculated. Parameters were compared between time points using the Wilcoxon signed-rank test. P-value of 0.05 was considered indicative of statistical significance.

\section{RESULTS}

The fused levels represented in the present study were L1-2 $(\mathrm{n}=1), \mathrm{L} 2-3(\mathrm{n}=3), \mathrm{L} 3-4(\mathrm{n}=3), \mathrm{L} 4-5(\mathrm{n}=9)$, and L5-S1 $(\mathrm{n}=1)$ (Table 1). The mean surgical time was $123.0 \pm 32.5$ minutes, and the mean blood loss was $387 \pm 95 \mathrm{~mL}$. No blood transfusion was required in any case.

\section{Lumbar Lordotic and Scoliotic Angles and Disc Space Height}

Although insignificant, the lumbar lordotic angle increased from $32.5 \pm 10.5^{\circ}$ (range, $25.8-47.0^{\circ}$ ) preoperatively to $34.0 \pm$ $12.3^{\circ}$ (range, 26.0-41.0) 12 months postoperatively. The scoliotic angle of the fused level improved from $11.5 \pm 6.0^{\circ}$ (range, 
Table 1. Patients' demographic data

\begin{tabular}{cccclcc}
\hline \hline Case & Age/sex & Fused level & BMD & \multicolumn{1}{c}{ Disease } & Complication & Results \\
\hline 1 & $35 / M$ & $L 4-5$ & 0.3 & Recurrent disc & - & Excellent \\
2 & $42 / F$ & $L 4-5$ & -0.5 & Recurrent disc & Dural tear & Excellent \\
3 & $35 / M$ & $L 4-5$ & -1.3 & Foraminal stenosis & - & Excellent \\
4 & $47 / M$ & $L 2-3$ & -1.2 & Upper lumbar disc & - & Excellent \\
5 & $68 / M$ & $L 4-5$ & -1.1 & Foraminal stenosis & - & Good \\
6 & $32 / F$ & $L 4-5$ & -0.5 & Foraminal stenosis & - & Excellent \\
7 & $47 / F$ & $L 1-2$ & -0.9 & Upper lumbar disc & - & Excellent \\
8 & $41 / M$ & $L 5-S 1$ & -0.5 & Foraminal stenosis & - & Excellent \\
9 & $39 / M$ & $L 2-3$ & -0.5 & Upper lumbar disc & - & Excellent \\
10 & $42 / F$ & $L 3-4$ & -0.7 & Foraminal stenosis & - & Excellent \\
11 & $54 / M$ & $L 3-4$ & -1.0 & Upper lumbar disc & Superficial infection & Good \\
12 & $50 / M$ & $L 4-5$ & -1.2 & Foraminal stenosis & - & Good \\
13 & $29 / M$ & $L 2-3$ & 0.5 & Upper lumbar disc & - & Excellent \\
14 & $43 / F$ & $L 4-5$ & -0.9 & Recurrent disc & - & Excellent \\
15 & $41 / M$ & $L 3-4$ & 0.5 & Upper lumbar disc & - & Excellent \\
16 & $42 / F$ & $L 4-5$ & -1.2 & Foraminal stenosis & - & Good \\
17 & $49 / M$ & $L 4-5$ & -1.4 & Foraminal stenosis & - & Excellent \\
\hline
\end{tabular}

$\mathrm{BMD}$, Bone mineral densitometry.

Table 2. Patients' radiological data

\begin{tabular}{lcccc}
\hline \hline & Preoperative & 3 months & 12 months & $\begin{array}{c}\text { p-value } \\
\text { (preoperative versus 12 months) }\end{array}$ \\
\hline Lordosis $\left(^{\circ}\right)$ & $32.5 \pm 10.5$ & $34.8 \pm 14.2$ & $34.0 \pm 12.3$ & $\mathrm{p}>0.05$ \\
Scoliosis $\left(^{\circ}\right)$ & $11.5 \pm 6.0$ & $10.6 \pm 5.6$ & $10.5 \pm 7.5$ & $\mathrm{p}>0.05$ \\
Disc space height $(\mathrm{mm})$ & $7.1 \pm 3.0$ & $9.6 \pm 3.0$ & $9.2 \pm 2.5$ & $\mathrm{p}=0.005$ \\
\hline
\end{tabular}

5-14) preoperatively to $10.5 \pm 7.5^{\circ}$ (range, 6-16) 12 months postoperatively; though, this change was also statistically insignificant. The disc space height increased from $7.1 \pm 3.0 \mathrm{~mm}$ preoperatively to $9.6 \pm 3.0 \mathrm{~mm}$ at the 3 -month postoperative examination but dropped to $9.2 \pm 2.5 \mathrm{~mm}$ at the 12 -month postoperative examination. Both the 3 and 12 month values were significantly different from the preoperative value (Table 2).

\section{Stability or Fusion}

At the 12-month postoperative examination, none of the 17 patients appeared to have fusion failure as defined by the presence of a definite radiolucent line around a cage or pedicle screws or more than $5^{\circ}$ of motion on dynamic flexion-extension views. No broken screw, screw loosening, or significant cage migration or subsidence was observed in any case.

\section{Clinical Outcomes}

As of 12 months postoperatively, all patients had achieved an Excellent or Good outcome (Excellent in 13 patients and Good in 4). The mean pain score was 7.5 prior to surgery and

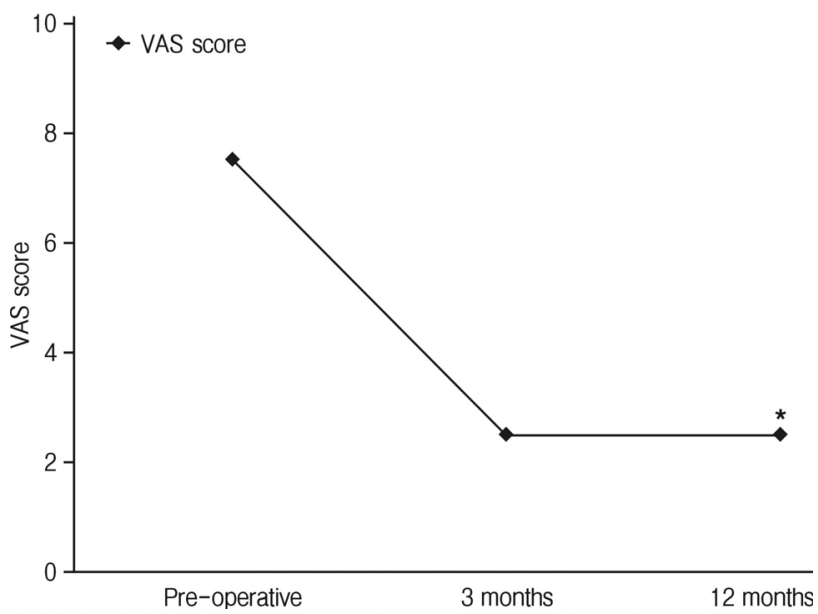

Fig. 2. Improvement in the pain score.

${ }^{*} p<0.001$ (preoperative versus 12 months). VAS, visual analogue scale.

had decreased to 2.5 at the 3-month postoperative examination; the decrease was maintained as of 12 months postoperatively (Fig. 2). No significant complication or neurological deterio- 

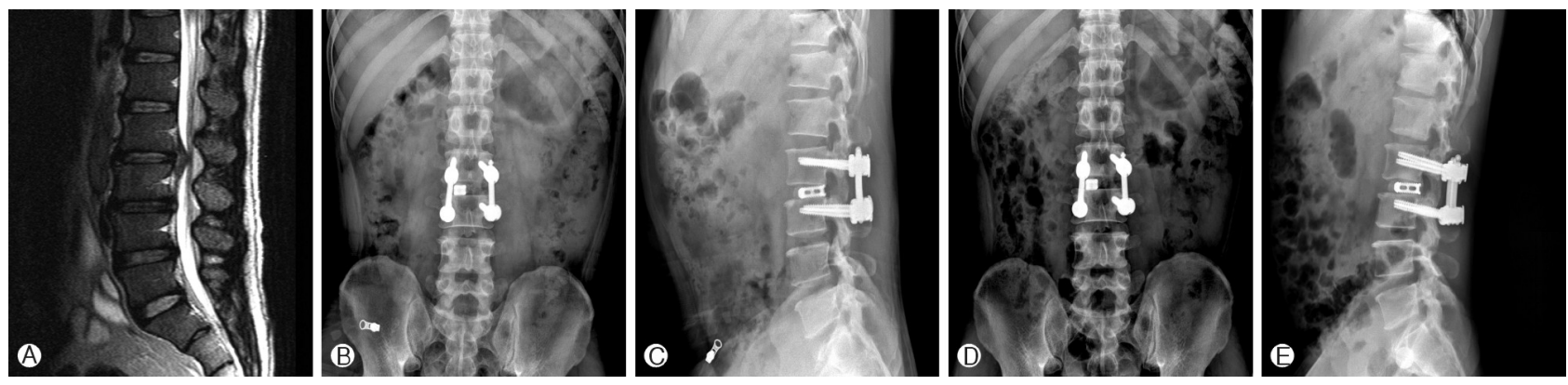

Fig. 3. A 47-year-old male patient with upper lumbar disc herniation at the L2-3 level. (A) Preoperative T2-weighted magnetic resonance image showing disc herniation compressing the dural sac. (B \& C) Simple radiographs taken 3 months after surgery demonstrate a single interbody cage with bilateral percutaneous screw fixation. (D\&E) Simple radiographs taken 12 months after surgery show no evidence of aggravated scoliosis, fusion failure or cage subsidence.
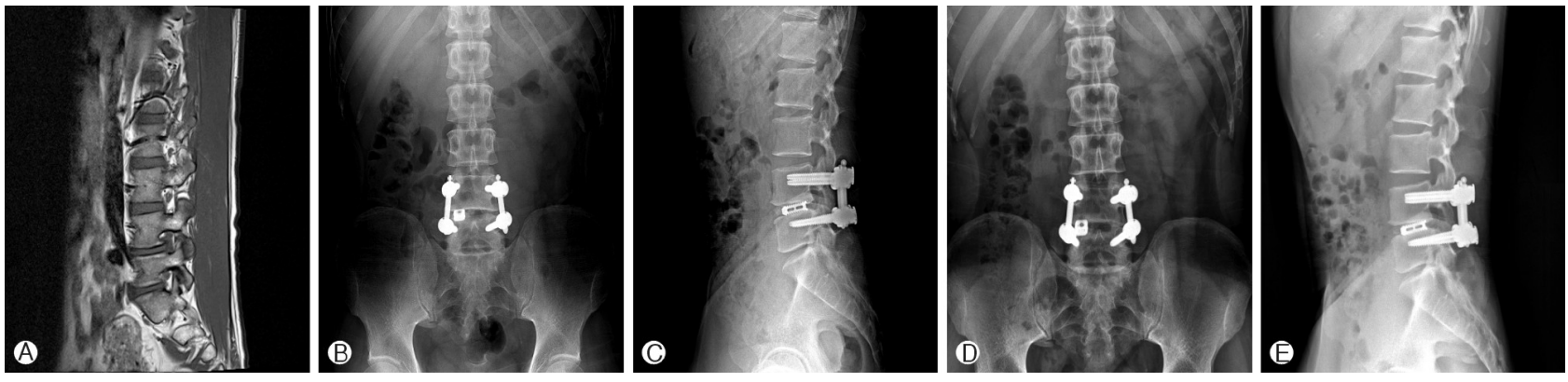

Fig. 4. A 35-year-old female patient with right foraminal stenosis at L4-5 level. (A) Preoperative T1-weighted magnetic resonance image shows severe right side foraminal stenosis at L4-5 level. (B \& C) Simple radiographs taken 3 months after surgery demonstrate a single interbody cage with bilateral percutaneous screw fixation. (D\&E) Simple radiographs taken 12 months after surgery show no evidence of aggravated scoliosis, fusion failure or cage subsidence.

ration occurred during the 12 months' follow-up (Fig. 3, 4).

\section{DISCUSSION}

PLIF has been associated with improvement of the fusion rate in combination with restoration of disc height and maintenance of vertebral alignment. Because the anterior and middle spinal columns support approximately $80 \%$ of the spinal load, placing the bone graft in this load-bearing position subjects it to compressive forces that enhance bone fusion ${ }^{2)}$. PLIF involving bilateral cages with pedicle screws has been recommended for routine use; in comparison with posterolateral instrumented fusion, it produces a significantly stiffer construct that protects the posterior instrumentation from failure and provides a circumferential fusion mass with an increased rate of successful fusion ${ }^{7}$. However, despite the satisfactory fusion rate, the bilateral cage PLIF procedure itself has some drawbacks. The massive skin incision and destruction of the posterior segments increase the risk for intraoperative blood loss and postoperative back pain. In addition, the procedure carries the risk of introducing bilateral nerve root injury or dural tearing because of the substantial retraction of the nerve roots required to gain access to the disc space. This is of special concern in patients with unilateral radiculopathy, in whom minimal nerve root manipulation on the contralateral asymptomatic side should be considered. A few authors to date have described the outcomes of unilateral cage insertion using bilateral pedicle screws. Molinari et al. ${ }^{5}$ reported that the unilateral cage produced good results and that patients with a unilateral cage experienced equal fusion and clinical success as those with bilateral cages. Fogel et al. ${ }^{1)}$ and Zhao et al. ${ }^{9}$ also reported acceptable results for PLIF with unilateral cage insertion.

The advantages of unilateral cage PLIF over standard PLIF using bilateral cages include lower risks of epidural fibrosis and injury to neural structures related to excessive root retraction ${ }^{4,5)}$. In addition, it can reduce blood loss as well as decrease cost by reducing the requirements for cages and transfusions. However, unilateral cage PLIF should not be considered for routine use if it lowers the fusion rate or exacerbates scoliotic deformity. Therefore, it is important to identify any negative effects on stability or spinal alignment. In our study, PLIF with a unilateral cage provided stability without scoliotic de- 
formity or subsidence. We had postulated that adequate stability could be achieved by interbody fusion using an inserted bone graft, rigid pedicle screws, and a rod system. Indeed, the central portion and a portion of the opposite disc space were curetted and adequate bone graft was inserted into the disc space even in the unilateral procedure. Moreover, pedicle screws and rods in addition to cage and bone graft insertion provided spinal stability sufficient to prevent scoliosis at the level of fixation. Bone grafting of the available surface area of the disc space is important for successful fusion. The surface area of exposed bone graft in a single cage is $138 \mathrm{~mm}^{2}$, whereas the surface area of a typical L5 lumbar endplate is 1,259 $\mathrm{mm}^{2}$. Therefore, a single cage will occupy only $10 \%$ of the endplate, and additional bone grafting to fill all available surface area is recommended ${ }^{1}$. The placement of additional bone graft material around the single cage may account for our undiminished rate of fusion success. Although our acceptable clinical and radiological results indicate that unilateral and bilateral PLIF are equivalent treatments, we must remember that there will be cases in which one is generally to be preferred over the other. For example, in patients with unilateral radiculopathy and acceptable bone mineralization, a unilateral approach allows for neural decompression of the symptomatic side and interbody stabilization. However, in patients with higher-grade spondylolisthesis, the bilateral approach provides continued intervertebral distraction while the disc space preparation and cage placement proceed on the contralateral side. This study has certain limitations that warrant discussion. First, the fusion status could not be determined accurately. A 12month follow-up period is relatively short for evaluation of spinal fusion status. Evaluation of intervertebral disc space fusion is difficult in the presence of a titanium cage because the titanium can obscure the presence of bridging bone. We evaluated the success of fusion by the amount of motion on flexion-extension radiographs. Second, we did not include patients with spondylolisthesis or osteoporosis. In such cases, we believe the insertion of bilateral cages should be considered in order to obtain more rigid fusion. Nevertheless, we believe this study serves as useful confirmation that PLIF with unilateral cage insertion can effectively provide stability and a successful clinical outcome when augmented with percutaneous screw fixation and insertion of sufficient bone graft material into the disc space. Randomized comparative clinical trials with longer observation periods in larger populations are necessary to confirm our results.

\section{CONCLUSION}

In patients with unilateral radiculopathy and acceptable bone mineralization, unilateral cage insertion followed by compact bone chip packing and bilateral percutaneous screw fixation can save time while providing good clinical and radiological outcomes.

\section{ACKNOWLEDGEMENT}

This study was supported by research funds from Chosun University grant 2007.

\section{REFERENCES}

1. Fogel GR, Toohey JS, Neidre A, Brantigan JW: Is one cage enough in posterior lumbar interbody fusion: a comparison of unilateral single cage interbody fusion to bilateral cages. J Spinal Disord Tech 20:60-65, 2007

2. Khoo LT, Palmer S, Laich DT, Fessler RG: Minimally invasive percutaneous posterior lumbar interbody fusion. Neurosurgery 51:166-181, 2002

3. Kim HS, Park KH, Ju CI, Kim SW, Lee SM, Shin H: Minimally Invasive Multi-Level Posterior Lumbar Interbody Fusion Using a Percutaneously Inserted Spinal Fixation System: Technical Tips, Surgical Outcomes. J Korean Neurosurg Soc 50:441-445, 2011

4. Lee JH, Lee JH, Yoon KS, Kang SB, Jo CH: Comparative study of unilateral and bilateral cages with respect to clinical outcomes and stability in instrumented posterior lumbar interbody fusion. Neurosurgery 63(1):109-113, 2008

5. Molinari RW, Sloboda J, Johnstone FL: Are 2 cages needed with instrumented PLIF ? A comparison of 1 versus 2 interbody cages in a military population. Am J Orthop 32:337-343, 2003

6. Mummaneni PV, Haid RW, Rodts GE: Lumbar interbody fusion: state of the art technical advances. Invited submission from the joint section meeting on disorders of the spine and peripheral nerves, March 2004. J Neurosurg Spine 1:24-30, 2004

7. Wang JC, Munnaneni PV, Haid RW: Current treatment strategies for the painful lumbar motion segment: posterolateral fusion versus interbody fusion. Spine 30:33-43, 2005

8. Yoon WS, Cho KS, Park JH, Park CK, Yoo DS, Huh PW, et al: Comparison of clinical outcomes: Posterolateral fusion versus posterior lumbar interbody fusion in low-grade degenerative spondylolisthesis. Korean J Spine 1(4):481-488, 2004

9. Zhao J, Wang X, Hou T, He S: One versus two BAK fusion cages in posterior lumbar interbody fusion to L4-L5 degenerative spondylolisthesis: A randomized, controlled prospective study in 25 patients with minimum two-year follow-up. Spine 27:27532757, 2002 